\section{OP0176 RNA SEQUENCING DETECTION OF GENE DYSREGULATION IN B CELLS SORTED FROM SALIVARY GLAND TISSUE AND PERIPHERAL BLOOD REVEALS NEW PATHWAYS INVOLVED IN SJÖGREN'S SYNDROME PATHOPHYSIOLOGY}

E. Rivière ${ }^{1}$, N. Tchitchek ${ }^{1}$, G. Nocturne ${ }^{1,2}$, J. Pascaud ${ }^{1}$, S. Boudaoud ${ }^{1}$, A. Thai ${ }^{3}$,

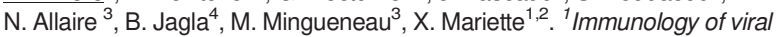
Infections and Autoimmune Diseases, IDMIT, CEA - Université Paris Sud INSERM U1184, Le Kremlin Bicêtre and Fontenay aux Roses; ${ }^{2}$ Rheumatology, Université Paris Sud, Le Kremlin Bicêtre, France; ${ }^{3}$ Immunology Research, Biogen, Cambridge, USA; ${ }^{4}$ Biomarker Discovery Platform UtechS CB, Hub de Bioinformatique et biostatistique C3IB, Institut Pasteur, Paris, France

Background: Primary Sjögren's syndrome (pSS) is a chronic auto-immune disorder characterised by lymphocytic infiltrates and destruction of the salivary glands (SG). Chronic B cell activation, the secretion of autoantibodies and the critical role of BAFF have been demonstrated. However, mechanisms leading to B cells dysregulation remain partially understood.

Objectives: To establish transcriptomic maps of the B cells sorted from the SG and from blood using RNASeq analysis.

Methods: Patients had pSS according to 2016 EULAR/ACR criteria and controls had sicca symptoms without any antibodies and with normal SG biopsy. B cells were sorted from SG biopsies and from blood using a FACS ARIA. Total RNASeq profiling was performed using MiSeq (lllumina). Statistical analysis (DESeq2) identified differentially expressed genes between pSS and controls in B cells sorted from SG (9 pSS and 4 controls), from blood (16 pSS and 7 controls); and between $B$ cells sorted from $S G$ and blood in the same patients (4 pSS). Functional enrichment analysis was performed using Ingenuity Pathway Analysis. Results: The pSS vs controls comparison in B cells sorted from SG identified upregulated genes involved in activation of $B$ cells including CD48, CD22 and CD40. TLR10, which is involved in innate immunity was also up-regulated in pSS. The analysis of the non-coding expressed RNAs showed an up-regulation of Mir155 which is essential for $B$ cell differentiation and antibody production (table $1 A$ ). In B cells sorted from blood, TLR7 and the downstream signalling molecule IRF7 were up-regulated in pSS. Additionally, IL-6 which is involved in B cells growth was up-regulated (table 1B). Enrichment analysis highlighted EIF2 signalling pathway, interferon (IFN) signalling pathway and role of JAK in IFN signalling. The paired comparison between B cells from SG and from blood identified up regulated genes including CD138, a plasma cell marker, IL-6, TLR5 and IFN induced genes (table 1C). Mir155HG was also up-regulated.

These results need to be confirmed by RT-qPCR and additional analysis of the non-coding expressed RNA is ongoing.

Table 1 Selection of genes differentially expressed between pSS and controls in $B$ cells sorted from biopsy (1A), blood (1B) and between SG and blood B cells from the same pSS patients (1C)

\begin{tabular}{cccc}
\hline & $\begin{array}{c}\text { Gene } \\
\text { Symbol }\end{array}$ & log2 fold-change & p-value \\
\hline 1A & \multicolumn{3}{c}{ Salivary B cells (pSS vs controls) } \\
\cline { 2 - 4 } & CD48 & 2.59 & 0.009 \\
\cline { 2 - 4 } & CD22 & 2.29 & 0.048 \\
\cline { 2 - 4 } & CD40 & 2.64 & 0.017 \\
\cline { 2 - 4 } & TLR10 & 5.67 & 0.002 \\
\cline { 2 - 4 } & Mir155HG & 4.94 & 0.002 \\
\hline 1B & \multicolumn{3}{c}{ Blood B cells (pSS vs controls) } \\
\cline { 2 - 4 } & TLR7 & 1.40 & 0.008 \\
\cline { 2 - 4 } & IRF7 & 0.76 & 0.041 \\
\cline { 2 - 4 } & IL-6 & 1.54 & 0.006 \\
\hline 1C & \multicolumn{3}{c}{ Salivary vs blood B cells in pSS } \\
\cline { 2 - 4 } & CD138 & 6.92 & $9.63 \mathrm{e}-05$ \\
\cline { 2 - 4 } & IL-6 & 3.05 & 0.004 \\
\cline { 2 - 4 } & TLR5 & 8.86 & 0.011 \\
\cline { 2 - 3 } & Mir155 & 3.10 & 06 \\
\hline
\end{tabular}

Conclusions: This study allowed exploring the mechanisms that support B cell activation in pSS focusing on tissue resident and circulating cells. Our data confirmed the $B$ cell activation and differentiation through several markers including $\mathrm{CD} 40, \mathrm{CD} 22, \mathrm{CD} 48, \mathrm{CD} 138$ and highlighted the role of innate immunity with the TLRs and key pathways including IFN and JAK signalling. Lastly, the role of an epigenetic regulation of $\mathrm{lg}$ secretion is suggested in tissue infiltrating B cells through mir155 expression. Precise understanding of these dysregulation should offer development of new targeted therapeutic perspectives for patients.

Acknowledgements: Arthritis Fondation Courtin for providing a PhD fellowship Disclosure of Interest: None declared
DOI: 10.1136/annrheumdis-2018-eular.3315

\section{OP0177 BCL6 IDENTIFIES ECTOPIC GERMINAL CENTRES IN SALIVARY GLAND BIOPSIES IN PRIMARY SJÖGREN'S} SYNDROME PATIENTS

U. Nakshbandi ${ }^{1}$, E.A. Haacke ${ }^{1,2}$, H. Bootsma ${ }^{1}$, F.K.L. Spijkervet ${ }^{3}$, A. Vissink ${ }^{3}$, B. van der Vegt ${ }^{2}$, F.G.M. Kroese ${ }^{1} .{ }^{1}$ Department of Rheumatology and Clinical Immunology, ${ }^{2}$ Department of Pathology and Medical Biology; ${ }^{3}$ Department of Oral and Maxillofacial Surgery, University of Groningen, University Medical Center Groningen, Groningen, Netherlands

Background: Primary Sjögren's syndrome (pSS) patients who exhibit germinal centres (GCs) within salivary gland parenchyma have higher focus scores and present with more disease activity than GC negative pSS patients. Moreover presence of GCs might be of clinical importance for stratification of treatment. However, there is considerable heterogeneity in reported findings concerning the presence of GCs. This can partially be explained by the difficulty in identifying GCs in diagnostic $\mathrm{H}$ and $\mathrm{E}$ sections and the lack of uniform histopathological criteria.

Objectives: The aim of this study was to assess the most appropriate way to identify unequivocally GCs in parotid and labial gland biopsies of pSS patients.

Methods: As part of routine diagnostic work-up for pSS, both parotid and labial salivary gland biopsies were taken from 100 consecutive patients suspected for pSS. Forty-two patients were classified as having pSS according to the ACREULAR criteria, the remaining 58 patients were classified as non-pSS sicca patients. Diagnostic salivary gland biopsies were formalin fixed, paraffin embedded and serially sectioned at 3-4 $\mu \mathrm{m}$ thickness. Sections were stained with $\mathrm{H}$ and $\mathrm{E}$ as well as immunohistochemically for CD3, CD20, CD21, CD45, $\mathrm{Ki} 67$ and Bcl6. Presence of GCs, the number of $\mathrm{GCs} / \mathrm{mm}^{2}$ salivary gland parenchyma and level of lymphoid organisation were determined in all sections.

Results: According to diagnostic $\mathrm{H}$ and $\mathrm{E}$ staining, in $15 \%$ and $2 \%$ of pSS patients GCs were present in parotid and labial salivary glands, respectively. Staining for the proliferation marker Ki67 and the GC-B cell associated transcription factor Bcl6, showed higher percentages: $23 \%$ and $25 \%$. Much higher percentages of follicular dendritic cell (FDCs) networks were revealed by CD21L $(45 \%$ and $55 \%$, for parotid and labial glands, respectively) compared to the number of GCs seen in tissue sections stained for Bcl6. Similarly, the median number of $\mathrm{CD} 21^{+} \mathrm{FDC}$ networks $/ \mathrm{mm}^{2}$ was significantly higher than the number of $\mathrm{GCs} / \mathrm{mm}^{2}$ as revealed by $\mathrm{H}$ and E, Ki67 and Bcl6. Careful evaluation of the consecutive sections stained for CD21L and Bcl6, showed that only roughly half of the FDC networks, also harbour GCs. Finally, not all sections that showed clearly defined GCs by Bcl6 staining, also revealed GCs by Ki67 or $\mathrm{H}$ and $\mathrm{E}$ staining.

Conclusions: Due to the difficulty in $\mathrm{GC}$ recognition, use of diagnostic $\mathrm{H}$ and $\mathrm{E}$ leads to an underestimation and incorrect identification of GCs, while using antiCD21L overestimates GC counts. This suggests that, although CD21 ${ }^{+}$FDC networks play an essential role in GC development and T/B cell compartmentalization, positivity for $\mathrm{CD} 21^{+}$FDC networks does not always imply presence of ectopic GCs. Furthermore, since Ki67 is an excellent marker for solely the dark zone of GCs, small GCs can be overlooked while other proliferative areas might be mistakenly identified as GCs. This study shows that staining for Bcl6 allows easy and unequivocal identification of GCs and should therefore be implemented in the histopathological evaluation of salivary gland biopsies of pSS patients.

Disclosure of Interest: None declared

DOI: 10.1136/annrheumdis-2018-eular.3741

\section{OP0178 ANALYSIS OF B-CELLS SUBSETS IN FIRST DEGREE RELATIVES OF PATIENTS WITH SYSTEMIC LUPUS ERYTHEMATOSUS}

A. Ruiz Roman ${ }^{1}$, A. Muñoz Jimenez ${ }^{1}$, J.M. Lucena ${ }^{2}$, M.A. Montes Cano ${ }^{2}$ B. Sanchez Sanchez ${ }^{2}$, N. Garrido Puñal ${ }^{1}$, E. Blanco Alonso ${ }^{1}$, R. Gill ${ }^{1}$, J. Quijada Carrera ${ }^{1}$, E. Rubio Romero ${ }^{1}$, J. Povedano Gomez ${ }^{1}{ }^{1}$ Reumatologia; ${ }^{2}$ Inmunologia, Hospital Universitario Virgen del Rocio, Sevilla, Spain

Background: Systemic lupus erythematosus (SLE) is an autoimmune, multiorgan disease characterised by periods of activity and remission. In lupus, one of the fields that has most helped his knowledge is the study of lymphocyte subpopulations through flow cytometry. Specifically, in the SLE some alterations have been detected at the level of $B$ lymphocytes as the increase of B-cells subsets such as plasmablasts, plasma cells, transitional cells. There are no studies to date that have analysed the behaviour of $B$ cells subsets in first-degree relatives of patients affected by lupus

Objectives: To analyse if there quantitative difference in the B-cells subsets of the first-degree relatives of SLE with respect to the control population (healthy) and the lupus population 
Methods: Transversal descriptive study. We included 13 patients diagnosed with SLE according to the criteria of the American College of Rheumatology (ACR) with positivity for antinuclear antibodies (ANA) and anti-DNA, 34 first-degree relatives and 50 healthy controls between the months of May 2016 and March 2017 None of the subjects evaluated received treatment with rituximab or belimumab. We analysed B-cells subsets (negative double, naïve B-cells, unswitched memory B-cells, switched memory B-cells) in all the subjects included in our study. The $95 \%$ confidence intervals were obtained for both the means and the percentage difference. The level of statistical significance was established at $p<0.05$. The data was analysed with IBM SPSS software Statistics 19 and EPIDAT 4.1.

Results: 47 subjects were analysed between relatives and patients, of which 33 ( $70.20 \%$ ) were women. 13 subjects (27.70\%) were diagnosed with lupus. $100 \%$ of those diagnosed with lupus were women. The mean $(\mathrm{X})$ and confidence intervals $(95 \% \mathrm{Cl})$ for the different subgroups (healthy subjects, subjects diagnosed with SLE, relatives of the first degree) is shown in table 1. In none of the subpopulations analysed in patients diagnosed with SLE in front of relatives of 1 st grade it has reached statistical significance. When analysing $B$ cells subsets of the three groups of subjects, we did find statistically significant differences between unswitched memory $B$ cells of healthy subjects and 1 st degree relatives (being lower in the group of healthy subjects) This finding has not been described in any previous study, although it should be noted that the sample before us is small.

Abstract OP0178 - Table 1

\begin{tabular}{lcccc}
\hline B-CELLS SUBSETS & & HEALTHY & SLE & RELATIVES \\
\hline Naïve (\%) & Mean & $70,4(95 \% \mathrm{Cl}:$ & $69,3(95 \% \mathrm{Cl}:$ & $65,8(95 \% \mathrm{Cl}$ : \\
& $(\mathrm{Cl} 95 \%)$ & $68,2$ to 72,7$)$ & $62,2$ to 76,3$)$ & $60,4$ to 71,2$)$ \\
\hline Doubles Negative & Mean & $4,1(95 \% \mathrm{Cl}: 3,5$ & $6,2(95 \% \mathrm{Cl}: 4,8$ & $2,9(95 \% \mathrm{Cl}: 1,9$ \\
(DN) (\%) & $(\mathrm{Cl} 95 \%)$ & to 4,6$)$ & to 7,6$)$ & to 4,0$)$ \\
Unswitched (\%) & Mean & $9,6(95 \% \mathrm{Cl}: 8,2$ & $13,0(95 \% \mathrm{Cl}: 7,4$ & $17,2(95 \% \mathrm{Cl}$ \\
& $(\mathrm{Cl} 95 \%)$ & to 11,1$)$ & to 18,7$)$ & $13,4$ to 21,1$)$ \\
Switched (\%) & Mean & $11,4(\mathrm{Cl} 95 \%$ & $11,5(95 \% \mathrm{Cl}: 6,3$ & $14,0(95 \% \mathrm{Cl}:$ \\
& $(\mathrm{Cl} 95 \%)$ & $=10,1-12,7)$ & to 16,7$)$ & $11,2$ to 16,8$)$ \\
\hline
\end{tabular}

Conclusions: There are quantitative differences between unswitched memory B-cells of healthy subjects and relatives of 1 st grade of SLE. More studies with a larger sample size are necessary to see the behaviour of the rest of B-type lymphocyte subpopulations

Disclosure of Interest: None declared

DOI: 10.1136/annrheumdis-2018-eular.7262

\section{OP0179 MOLECULAR DOCUMENTATION OF THE CLONAL EVOLUTION OF A DIFFUSE LARGE B-CELL LYMPHOMA OUT OF CLONALLY EXPANDED RHEUMATOID FACTOR-EXPRESSING B CELLS IN A SJÖGREN'S SYNDROME PATIENT}

R.J. Bende ${ }^{1}$, L.M. Slot ${ }^{1}$, T.A. Wormhoudt ${ }^{1}$, M.J. Kwakkenbos ${ }^{2}$, A.H. van Kampen ${ }^{3}$, A. Jongejan ${ }^{3}$, J.E. Guikema ${ }^{1}$, C.J. van Noesel ${ }^{1}{ }^{1}$ Pathology, Academic Medical Center, ${ }^{2}$ AIMM Therapeutics, AIMM Therapeutics; ${ }^{3}$ Clinical Epidemiology, Biostatistics and Bioinformatics, Academic Medical Center, Amsterdam, Netherlands

Background: Sjögren's syndrome (SS) is the autoimmune disease with the highest risk of lymphoma development. SS patients develop most frequently MALT lymphoma and to a lesser extent diffuse large B-cell lymphoma (DLBCL). Previously, we have shown that at least $40 \%$ of salivary gland MALT lymphomas express groups of near identical (also called stereotypic) B-cell receptors, which display in vitro mono-specific rheumatoid factor (RF) reactivity ${ }^{1}$. Recently, we have analysed the B-cell immunoglobulin heavy variable (IGHV) repertoire in 4 SS labial salivary glands. In 2 out of 4 salivary glands only one minor stereotypic RF B-cell clone was detected. Interestingly, in one salivary gland of patient SG22 a highly expanded stereotypic RF-expressing B-cell clone was present, which was also detected in peripheral blood. Twenty six months later, a clonally-related DLBCL was diagnosed ${ }^{2}$.

Objectives: To study the molecular alterations present in the RF-expressing $\mathrm{B}$ cell clones of salivary gland, peripheral blood and in the DLBCL of SS patient SG22.

Methods: From peripheral blood 4 RF-expressing immortalised B-cell clones with identical IGHV rearrangements were isolated. These 4 RF-clones were analysed by whole exome sequencing and the identified non-synonymous exome mutations were traced in the salivary gland and in the DLBCL, using targeted next generation sequencing.

Results: In total we identified 56 exome mutations in the 4 RF B-cell clones. Twelve non-synonymous mutations were shared between all 4 RF-clones, of which one was a missense mutation in CARD11, a well-known oncogenic mutation of DLBCL. In the salivary gland all shared 12 non-synonymous mutations were detected, whereas in the DLBCL only 4 of these mutations were detected.
Remarkably, the CARD11 mutation was not detected in the DLBCL. The exome mutations were analysed for typical characteristics of induction by the B-cell specific mutator AID. This revealed that the large majority of mutations $(80 \%)$ were (i) not located within $1,5 \mathrm{kB}$ of the transcription initiation site of the genes, (ii) did not concern C/G nucleotides in AID hotspots (WRC/GYW) and (iii) were not in genes expressed by B cells. This lack of evidence for AID involvement, suggests that the exome mutations of the RF-clones were due to replication errors, obtained during their clonal expansion. Interestingly, as compared to the RF-clones of peripheral blood, the DLBCL accumulated 44 extra somatic mutations together in IGHV and IGKV. As expected, these new IGHV/IGKV mutations showed the characteristics of AID involvement, indicating that in an ancestor RF-clone, AID activity was induced.

Conclusions: We have documented the clonal outgrowth and diversification of a RF-specific B-cell clone in a SS patient and the evolution of this clone into a DLBCL. Our analysis are indicative for AID activity in the lymphoma cells, represented by accumulation of IGHV/IGKV mutations. However, we have no evidence that AID is instrumental in the accumulation of the non-IG mutations in the RF clones from peripheral blood.

\section{REFERENCES:}

[1] Bende, et al. J. Exp. Med 2005;201:1229-1241.

[2] Bende, et al. Arthritis Rheumatol 2015;67:1074-1083.

Disclosure of Interest: None declared

DOI: 10.1136/annrheumdis-2018-eular.4401

\section{OP0180 TYPE I INTERFERON IS PRODUCED BY NON- HAEMATOPOIETIC TISSUE RESIDENT CELLS BUT NOT PDCS IN PRE-CLINICAL AUTOIMMUNITY AND SLE}

A Psarras $^{1,2}$, A. Alase ${ }^{1}$, A. Antanaviciute ${ }^{3}$, I. Carr ${ }^{3}$, M. Wittmann ${ }^{1}$, G. Tsokos ${ }^{2}$,

P. Emery ${ }^{1}$, E.M. Vital ${ }^{1} .{ }^{1}$ Leeds Institute of Rheumatic and Musculoskeletal Medicine, University of Leeds, Leeds, UK; ${ }^{2}$ Beth Israel Deaconess Medical Center, Harvard Medical School, Boston, USA; ${ }^{3}$ Leeds Institute for Data Analytics, University of Leeds, Leeds, UK

Background: Systemic Lupus Erythematosus (SLE) is characterised by persistently high type I interferon (IFN) activity. Plasmacytoid dendritic cells ( $p D C s$ ) pro duce large amounts of IFNs in viral infection, although these immunogenic properties are usually strictly regulated. In vitro, pDCs are responsive to nucleic acids and they have therefore been postulated to be the main source of type IFNs in SLE. However, their function is not fully established in human SLE.

Objectives: To investigate the dysregulated IFN axis in patients with pre-clinical autoimmunity and SLE.

Methods: Patients with SLE who met 2012 ACR/SLICC criteria were recruited. We also recruited healthy controls $(\mathrm{HC})$ and therapy-naïve individuals presenting with ANA and 1-2 clinical symptoms, but not meeting ACR/SLICC criteria, of whom $17 \%$ progressed to SLE (At-Risk). IFN activity was evaluated by measuring a score of IFN-responsive genes in the PBMCs using TaqMan. pDCs were immunophenotyped as well as studied in vitro for production of proinflammatory cytokines and induction of $\mathrm{T}$ cell responses using flow cytometry. pDCs were sorted and sequenced using high-sensitive RNA sequencing. IFN expression was visualised in skin biopsies using in situ hybridisation. Keratinocytes were isolated from fresh skin biopsies and cultured in vitro; IFN production was measured by qPCR and ELISA.

Results: Most of the SLE and At-Risk patients had increased IFN activity, which correlated with disease activity and clinical features. In contrast, circulating pDCs were decreased in both SLE and At-Risk patients and their numbers did not correlate with any clinical features or IFN status. In vitro stimulation revealed that $\mathrm{pDCs}$ from SLE and At-Risk patients could not produce IFN- $\alpha$ and TNF- $\alpha$ upon stimulation with TLR9 or TLR7 agonists. In addition, they induced significantly less T cell activation and proliferation compared to $\mathrm{HC}$ pDCs. RNA-seq data analysis showed an upregulation of IFN-responsive genes in most of the SLE and At-Risk pDCs but not transcripts of any IFN subtypes. Other upregulated pathways were related to immune regulation and senescence. Phenotypically, SLE pDCs were characterised by upregulation of regulatory receptors and increased telomeric erosion. In situ hybridization revealed high IFN expression in the epidermis but not in lymphocyte-infiltrating areas of lesional biopsies from SLE patients. High expression of IFN was also observed in epidermis of At-Risk individuals without any signs of cutaneous inflammation. In vitro stimulation of freshly isolated keratinocytes also showed a notable increase in IFN production.

Conclusions: In SLE, non-haematopoietic tissue resident cells are a dominant source of IFN and this is present prior to clinically overt disease. Meanwhile, the professional IFN-producing pDCs have lost their immunogenic properties. These findings suggest an important role for tissue resident cells in autoimmunity and may facilitate novel therapeutic interventions.

Disclosure of Interest: None declared 